\title{
Bürokratieabbau und Bürgervertrauen: Mahnworte an Landtagsabgeordnete
}

Blanke, Bernhard, Frank Nullmeier, Christoph Reichard und Göttrik Wewer (Hrsg.): Handbuch zur Verwaltungsreform, 4. Auflage, VS Verlag für Sozialwissenschaften, Wiesbaden 2011, 616 Seiten, $€ 49,95$.

Bürgerkommune, E-Government, Kommunalisierung, öffentlich-private Partnerschaften: Debatten um die Verwaltung in Gemeinden und Bundesländern haben ihre Begriffe wie auch Schwerpunkte in den vergangenen Jahren, teils in Wellenbewegungen, deutlich verändert. Sichtbar wird das in der vierten Auflage des „Handbuchs zur Verwaltungsreform“, das auf mehr als 600 Seiten Beamten und Politikern, vor allem aber Landtagsabgeordneten eine Handreichung gibt über Vorzüge und Nachteile dieser Reformvorschläge. Die vier Herausgeber um den hannoverschen Staatswissenschaftler Burkhard Blanke wollen das gesammelte „strategische“ Wissen über die Verwaltungsreform systematisch in einer Sprache erschließen, die überwiegend gut lesbar ist.

Bei aller Detailfülle - nicht jede Wissbegier wird erfüllt. So wird die politische Debatte um eine Rekommunalisierung der Versorgung etwa mit Strom kaum erwähnt. Im Abschnitt über Personal wird verglichen, dass in Großbritannien nur jeder zehnte Angestellte im öffentlichen Dienst Beamter ist, in den Niederlanden aber 100 Prozent (Deutschland liegt mit 37 Prozent an Beamten im unteren Mittelfeld); und dass bei der Aufgabenverteilung zwischen Zentralstaat und Kommunen Schweden „Europameister" bei der „echten“ Kommunalisierung ist (dort sind 83 Prozent aller Angestellten im öffentlichen Dienst für Kommunen tätig). Wie hoch der Anteil Angestellter im öffentlichen Dienst aber an allen Beschäftigten im jeweiligen Lande ist, fehlt.

Sechs Bundesländer haben in den vergangenen Jahren bei ihren Verwaltungsstrukturreformen einen ,großen Wurf" angestrebt: Nordrhein-Westfalen, Sachsen, Sachsen-Anhalt, Mecklenburg-Vorpommern und vor allem seit 2005 Baden-Württemberg und Niedersachsen. Hier mag das Buch den Landtagen wie auch den Innenministerien eine Handreichung bieten vor der Verabschiedung von Strukturreformgesetzen im Kleinen und Großen. Dort und auch in den Kommunen zeigt sich eine Vielfalt von Reformansätzen, die sich im Ausland widerspiegelt: Mehrheitlich bündeln und zentralisieren sie, um Kosten zu sparen, teilen aber auch auf, um bürgernäher zu sein. So haben sich gerade unitarisch-zentralistisch aufgebaute Staaten föderalisiert wie Spanien, Belgien und Großbritannien, oder zumindest an Regionen größere Befugnisse übertragen wie Frankreich und Schweden. In Deutschland wurden Kommunen und Landkreise zusammengelegt, ihnen dann aber mehr Kompetenzen übertragen.

Bei der Verwaltungsreform gibt es Stichworte, die fast allen Bundesländern gemein sind. Auf Entbürokratisierung stützt sich beständig fast jeder - so jede Regierungserklärung im Bundestag seit Willy Brandt, getragen von sechs Sachverständigen-Kommissionen in den vergangenen vier Wahlperioden. Der Erfolg ist überschaubar. Stärker wurde das Ziel einer Privatisierung staatlichen Eigentums und öffentlicher Aufgaben umgesetzt: Zwischen 1991 und 2007 sank die Zahl der Beschäftigten im öffentlichen Dienst des Bundes von 650.000 auf 470.000. Dabei dienen Privatisierungen dem Bürokratieabbau wie auch der Haushaltssanierung. Ohne eine Einbindung der Landtage und Begleitung durch sie an verschiedenen Stellen ist eine grundlegende Verwaltungsreform kaum denkbar - das reicht vom Gesetzesvorbehalt über die Gesetzesfolgenabschätzung bis zum Haushaltsrecht. 
Oft werden neben Auslagerungen oder Privatisierungen Zwischenformen gewählt mit öffentlich-privaten Partnerschaften in praktisch allen Feldern öffentlicher Leistungserbringung, derzeit bei gut fünf Prozent der staatlichen Bruttoinvestitionen mit Schwerpunkten im Hochbau (Schulen und Kindertagesstätten, Sport und Kultur, Verwaltung und Justiz). Bisweilen bringen sie Zwist, etwa zwischen Regierung und Opposition im Niedersächsischen Landtag zum geplanten Bau einer Justizvollzugsanstalt. Neun Bundesländer haben im vergangenen Jahrzehnt dafür Leitstellen, Netzwerke oder Zentren gegründet. Ob die Ziele - vor allem bessere Wirtschaftlichkeit - erreicht werden, hänge, so die Autoren, stark vom Vorgehen vor Ort und der handelnden Personen ab; generelle Einschätzungen seien schwierig.

Im Blick auf die Bahnhofsdebatte in Stuttgart bietet eine vergleichende Studie zum Vertrauen in die öffentliche Verwaltung in europäischen Ländern Hilfreiches, insbesondere auch für hellhörige Abgeordnete. Befragungen zeigen, dass unter fünf Industriestaaten den Vereinigten Staaten, Deutschland, Frankreich, Großbritannien und Schweden Deutschlands Verwaltung zwischen 1981 und 2007 stets das niedrigste Vertrauen genoss. Dabei ging die Schere auseinander. In Nordamerika und Deutschland sank es, in Schweden stieg es stark. So vertrauten dort zuletzt 66 Prozent der befragten Bürger ihrer Verwaltung, in Deutschland nur 31 Prozent. Warum das aber so ist, vermochte auch diese Studie nicht zuverlässig zu erklären. Hier wäre sicher interessant gewesen zu hören, ob es eine Korrelation gibt zwischen dem Vertrauen in die Verwaltung, in die Politik, und die Parlamente.

Robert von Lucius

\section{Abgeordnetenentschädigung: aktuell kommentiert}

Von Arnim, Hans Herbert und Thomas Drysch: Kommentierung des Art. 48 Grundgesetz, in: Rudolf Dolzer, Wolfgang Kahl und Christian Waldhoff (Hrsg.): Bonner Kommentar zum Grundgesetz, 149. Aktualisierung, C.F. Müller Verlag, Heidelberg Dezember 2010, 162 Seiten, $€$ 349,95 (gesamtes Loseblattwerk zzgl. Aktualisierungslieferungen).

Der Zwiespalt der Loseblatt-Kommentare besteht darin, dass die Kommentierungen einzelner Bestimmungen oft umfassend sind, ja monografisch. Aber genauso oft sind sie überaltert und damit für die Beantwortung aktueller Fragen kaum noch brauchbar. Art. 48 GG über die Entschädigung der Bundestagsabgeordneten im Bonner Kommentar hatte Hans Herbert von Arnim zuletzt 1980 bearbeitet. Die Bestimmung blieb seitdem zwar unverändert, aber ihre Anwendung in der Praxis des Bundestags hat immer wieder neue verfassungsrechtliche Fragen aufgeworfen; und nachdem das Bundesverfassungsgericht im so genannten ersten Diätenurteil aus dem Jahr 1975 Art. 48 GG auch zur Auslegung der Diätenbestimmungen eines Landesparlaments heranzog, gilt sie - zu Recht oder zu Unrecht - als Maßstab auch landes(verfassungs-)rechtlicher Diätenregelungen. Für die überfällige Neukommentierung hat von Arnim seinen ehemaligen Mitarbeiter und ausgewiesenen Steuerfachmann Thomas Drysch als Koautor gewonnen. Die Kommentierung ist wiederum umfassend, aber kaum umfangreicher als zuvor; der Zuwachs an Seiten ist in erster Linie dem lesefreundlichen Satzbild geschuldet. 